\title{
Development of visually evoked cortical activity in infant macaque monkeys studied longitudinally with fMRI
}

\author{
Zoe Kourtzi $^{\mathrm{a}, 1}$, Mark Augath ${ }^{\mathrm{a}}$, Nikos K. Logothetis ${ }^{\mathrm{a}}$, J. Anthony Movshon ${ }^{\mathrm{b}}$, Lynne Kiorpes ${ }^{\mathrm{b}, *}$ \\ ${ }^{a}$ Max Planck Institute for Biological Cybernetics, 72076 Tuebingen, Germany \\ ${ }^{\mathrm{b}}$ Center for Neural Science, New York University, New York, NY 10003, USA \\ Received 19 October 2005; accepted 2 December 2005
}

\begin{abstract}
We studied the development of visual activation longitudinally in two infant monkeys aged 103-561 days using the BOLD fMRI technique under opiate anesthesia and compared the results with those obtained in three adult animals studied under identical conditions. Visual activation in primary visual cortex, V1, was strong and reliable in monkeys of the youngest and oldest ages, showing that functional imaging techniques give qualitatively similar results in infants and adults. Visual activation in extrastriate areas involved in processing motion (MT/V5) and form (V4) was not evident in the younger animals, but became more adult-like in the older animals. This delayed onset of measurable BOLD responses in extrastriate visual cortex may reflect delayed development of visual responses in these areas, although at this stage it is not possible to rule out either effects of anesthesia or of changes in cerebral vascular response mechanisms as the cause. The demonstration of visually evoked BOLD responses in young monkeys shows that the BOLD fMRI technique can usefully be employed to address functional questions of brain development.
\end{abstract}

(C) 2006 Elsevier Inc. All rights reserved.

Keywords: Functional imaging; Visual development; Macaque monkeys; Visual cortex

\section{Introduction}

The process of brain development has long been an important focus of neuroscience research. Behavioral studies of newborn primate infants show significant postnatal development of sensory and cognitive function [1]. The relationship between structural and functional changes in the brain postnatally and changes in behavior remains poorly understood. Recent advances in functional magnetic resonance imaging techniques offer the opportunity to observe the maturation of cortical organization at the level of functional areas. Moreover, this relatively noninvasive technique permits longitudinal study of individuals, thus offering the opportunity for improving our understanding of sequential developmental events and directly correlating brain function and performance.

One of the best studied systems in the brain is the visual system. Substantial knowledge exists of the neural mechanisms limiting visual performance in infants [2,3]. However,

\footnotetext{
* Corresponding author. Fax: +1 2129954183.

E-mail address: lynne@cns.nyu.edu (L. Kiorpes).

${ }^{1}$ Present address: School of Psychology, University of Birmingham, Edgbaston, B15 2TT, Birmingham, UK.
}

current research shows that much of the important maturational events are likely to be beyond the primary visual cortex, V1, in extrastriate cortical visual areas such as V4 or MT/V5, or perhaps even visual association areas. Thus, an obvious and important question to address is, what is the sequence of development of higher visual cortical areas in relation to primary visual cortex? A few studies have attempted to investigate this question at the single-neurone level in nonhuman primates [4-8]. These studies show on balance later development of extrastriate and inferotemporal visual areas. Similar conclusions come from studies using the technique of 2-deoxyglucose autoradiography to measure visually evoked metabolic activity [9]. However, it is difficult to gain a sense for whether there is a clear hierarchical cascade, a differential development of ventral and dorsal stream areas [10], or perhaps a generalized poststriate maturation from such approaches.

A few investigations using fMRI techniques in human infants are beginning to address questions of global sequences of brain development $[11,12]$. Since much of the research characterizing anatomical and physiological maturation has been done in animal models such as cat and monkey, and quantitative longitudinal studies of visual 
development in monkeys exist [13-15], it is important to develop imaging technology to bring a more global perspective to this question. We have undertaken the study of postnatal maturation of early visual areas using fMRI. In this report, we describe technical aspects of this methodology and data suggesting late emergence of reliable BOLD activation in extrastriate compared to striate visual areas.

\section{Methods}

Experiments were conducted in infant and adult Macaca mulatta monkeys. The studies were approved by the local authorities (Regierungspräsidium) and were in full compliance with the guidelines of the European Community (EUVD 86/609/EEC) for the care and use of laboratory animals. We scanned two infant monkeys (aged 3.5 months at the start of the study) repeatedly at different ages (weight range from beginning to end of the study, $1.1-2.9 \mathrm{~kg}$ ). We obtained imaging data from seven sessions from one infant and four from another, during which the stabilization of the animals in the scanner was successful and their physiological condition stable. The ages of the infant monkeys during scanning ranged from 103 to 561 days. We also scanned three adult monkeys (age, 4.9-6.8 years; weight, 5.5$7.0 \mathrm{~kg}$ ) during the same period as the infant monkeys. One of the adult monkeys was scanned twice at different ages (4.9 and 6.8 years).

\subsection{Animal preparation and maintenance}

The handling and anesthesia protocol used ensure stressfree treatment of the animal. The anesthesia protocol for imaging of the adult animals followed the one used in previous studies [16] and was adapted for imaging of the infant monkeys. Specifically, for the adult monkeys after premedication with glycopyrrolate $(0.01 \mathrm{mg} / \mathrm{kg}$, im) and ketamine $(15 \mathrm{mg} / \mathrm{kg}$, im), an intravenous catheter was inserted to the saphenous vein (20-gauge). Animals were preoxygenated and anesthesia was induced with fentanyl $(3 \mu \mathrm{g} / \mathrm{kg})$ and thiopental $(5 \mathrm{mg} / \mathrm{kg})$. Succinylcholine chloride $(3 \mathrm{mg} / \mathrm{kg})$ was used as a relaxant before intubation of the trachea. Following intubation, the animals were ventilated using a Servo Ventilator $900 \mathrm{C}$ (Siemens, Germany), maintaining an end-tidal $p \mathrm{CO}_{2}$ of $34-35 \mathrm{~mm}$ $\mathrm{Hg}$ and oxygen saturation over 95\%. Anesthesia was induced with isoflurane in air and maintained with remifentanil $\left(0.5-2 \mu \mathrm{g} \mathrm{kg}^{-1} \mathrm{~min}^{-1}\right)$. Muscle relaxation was achieved with mivacurium chloride $\left(5 \mathrm{mg} \mathrm{kg}^{-1} \mathrm{~h}^{-1}\right)$. Lactated Ringer's solution was given intravenously at a maximum rate of $10 \mathrm{~mL} \mathrm{~kg}^{-1} \mathrm{~h}^{-1}$. For the infant monkeys, the anesthesia protocol differed from that used for the adult animals in the following aspects: (a) a 24-gauge intravenous catheter was used; (b) no succinylcholine chloride was used before intubation and (c) the remifentanil and mivacurium chloride dosages were about one fifth of the adult dosage. Physiological parameters (heart rate, blood pressure, body temperature, blood oxygenation and expiratory $\mathrm{CO}_{2}$ ) were monitored continuously and maintained within normal limits. Recovery from anesthesia was achieved by withdrawing first the mivacurium chloride and then the remifentanil; alertness and normal respiration were typically restored after approximately $30-40 \mathrm{~min}$.
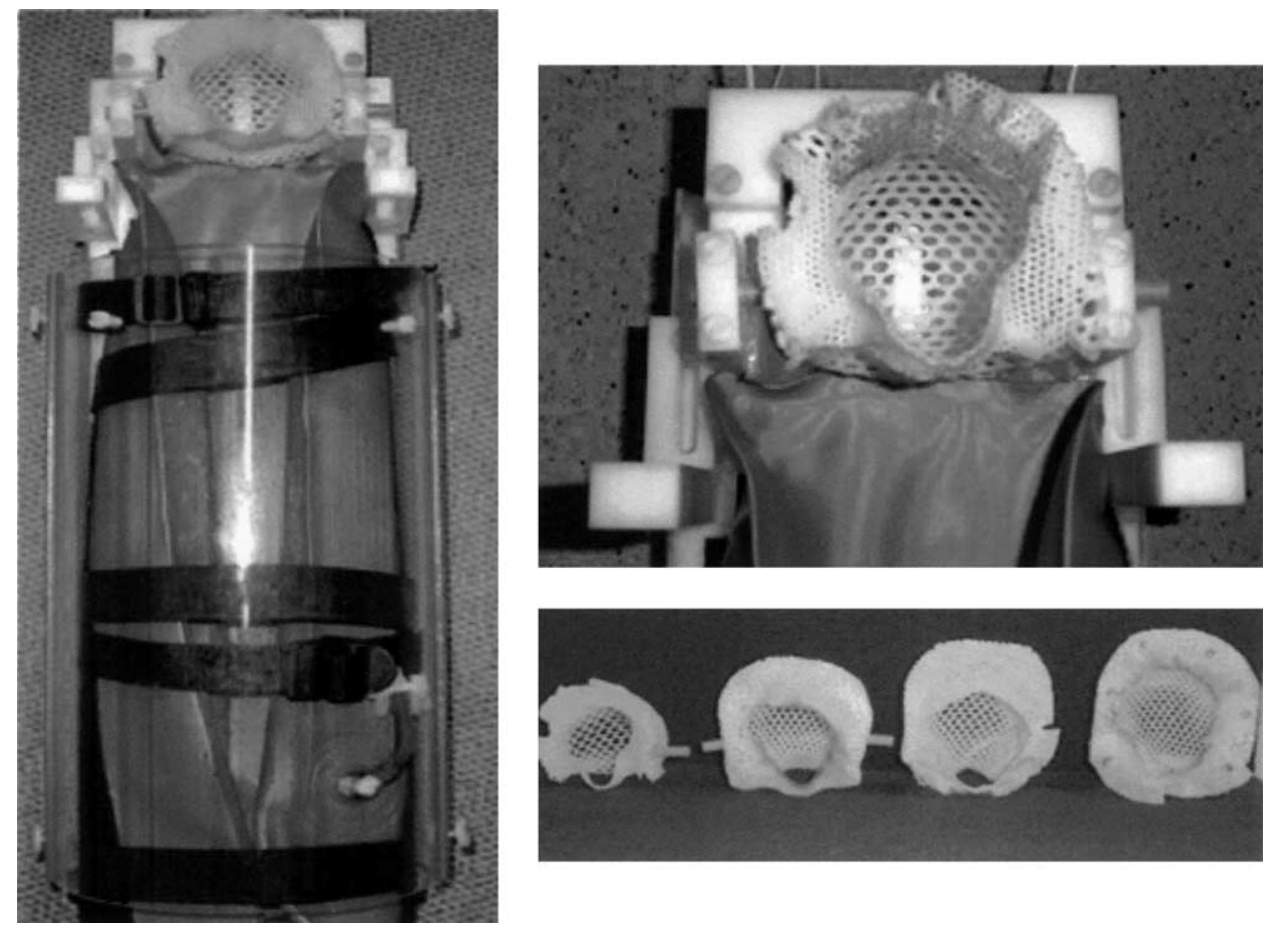

Fig. 1. (Left) The modified chair used to hold the infant monkeys during functional imaging. (Right) Views of the thermoplastic face moulds used to stabilize the head. 


\subsection{Visual stimuli and experimental design}

We used a $100 \%$ contrast rotating checkerboard polar pattern stimulus covering a large area of the central visual field $\left(30^{\circ}\right.$ horizontal $\times 23^{\circ}$ vertical, centered on the fovea), delivered binocularly through a fiber-optic imaging system (Avotec). The spatial frequency of the stimulus was $30^{\circ}$ per cycle and the temporal frequency of rotation $1 / 6 \mathrm{~Hz}$. We presented the rotating polar stimulus for $48 \mathrm{~s}$ followed by a 48-s background stimulation (gray full-field stimulus at the average luminance of the polar stimulus). The direction of rotation of the polar stimulus was reversed every $5 \mathrm{~s}$ from clockwise to counterclockwise. This stimulus-blank sequence was repeated four times within a scan for a total duration of $384 \mathrm{~s}$. We collected data from two to three scans in each session. This stimulus has previously been shown to elicit robust activation in multiple areas of the visual cortex of the anesthetized monkey [16]. The procedures for aligning the stimuli with the visual axes were as previously described [16]. Contact lenses with the appropriate dioptric power, as measured ophthalmoscopically and with a modified fundus camera after the introduction of paralysis for each scanning session, were used to make the animal's retinas conjugate with the visual stimuli.

\subsection{Imaging}

Experiments were conducted in a vertical 4.7-T scanner with a 40-cm-diameter bore (BioSpec 47/40v, Bruker Medical, Ettlingen, Germany). The system had a $50 \mathrm{mT} / \mathrm{m}$ (180-s rise time) actively shielded gradient coil (Bruker, B-GA 26) of $26 \mathrm{~cm}$ inner diameter. For the adult animals, we used a custom chair and custom system for positioning the monkey within the magnet [16]. For the infant monkeys,
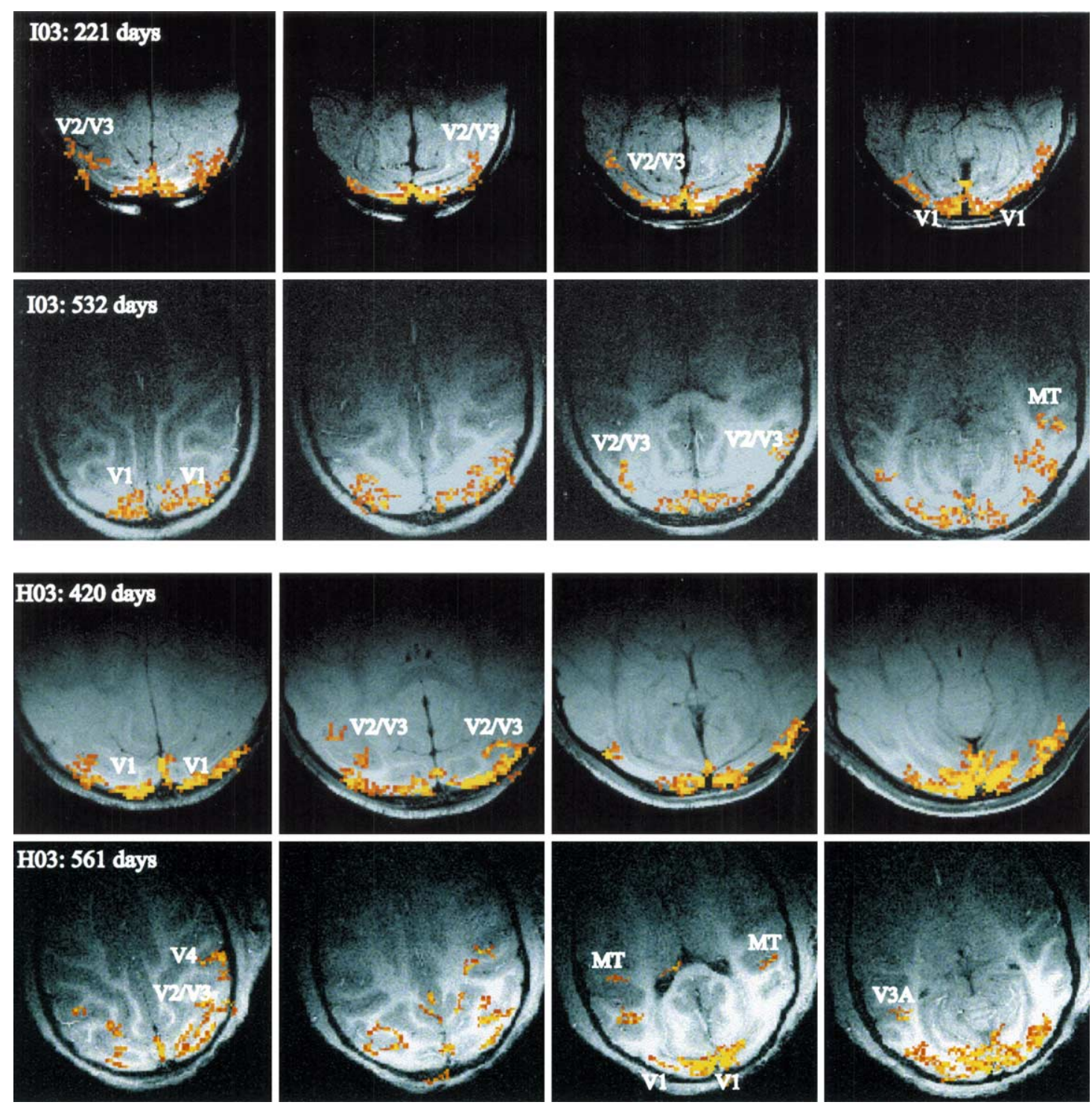

Fig. 2. Functional maps of visual activation in two infant monkeys, each shown at two different ages. Activations $[P$ (corrected) $<.001:$ yellow; $P$ (corrected) $<.05$ : red] are shown on four sequential slices (superior to inferior). Note the increased activation of areas outside V1 at older ages. 

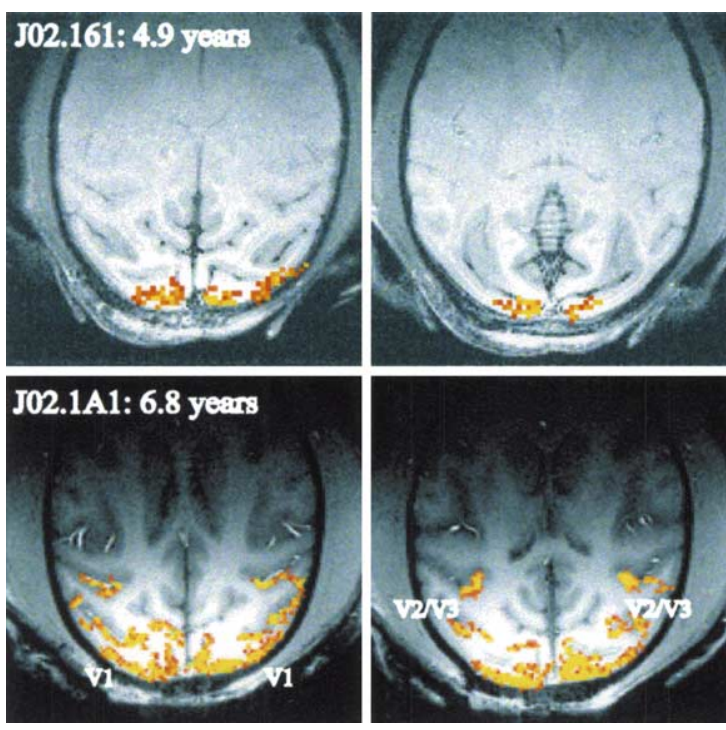
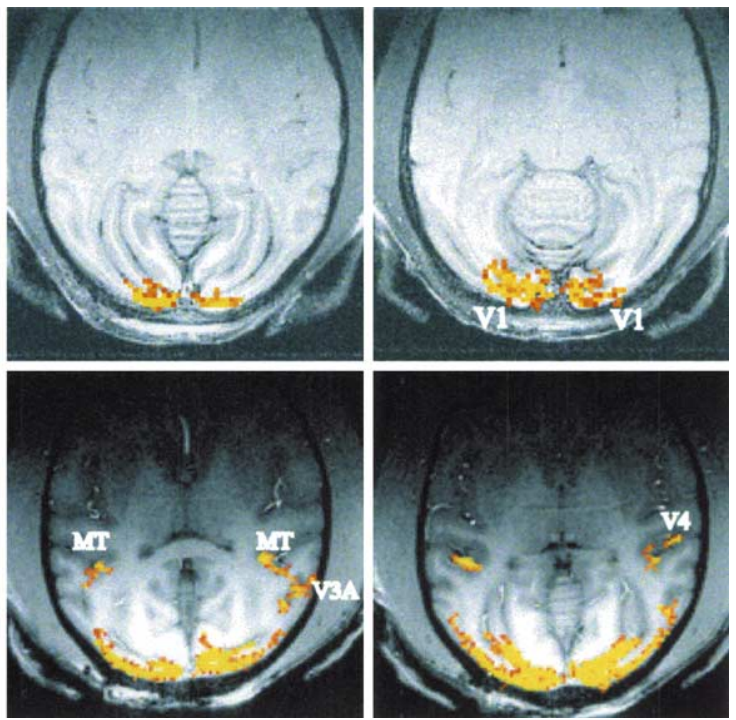

Fig. 3. Functional maps of visual activation in one adult monkey imaged at two ages.

we used the same system, but the animals were first positioned in a smaller chair that was then stabilized within this system (Fig. 1). Custom-made plastic head holders were used for stabilizing the adult animals in the scanner (surgical procedures as described previously [16]). The use of head holders for the infant monkeys would have required multiple surgeries due to continuous skull growth. To avoid this, we used head moulds from thermoplastic material (WFR/Aquaplast, Wycoff, NJ) that were prepared before every scanning session in the younger animals and a bite bar in the older ones. These procedures ensured adequate stabilization of the infants in the scanner without surgical intervention.

We collected 13-15 horizontal slices using multishot (8) GE-recalled EPI images with a $128 \times 128$ matrix $\left(1 \times 1 \mathrm{~mm}^{2}\right.$ resolution, slice thickness $2 \mathrm{~mm}, \mathrm{TE}=20 \mathrm{~ms}$, $\mathrm{TR}=750 \mathrm{~ms}, \mathrm{FA}=40^{\circ}$ ) using quadrature surface coils. Anatomical images were acquired using a matrix of
$256 \times 256(0.5 \times 0.5$-mm resolution, inversion recovery-rapid acquisition with relaxation enhancement).

\subsection{Data analysis}

MRI data were processed using the BrainVoyager 4.9 software. Preprocessing of all the functional data included temporal high-pass filtering and removal of linear trends in the BOLD signal. For each session, we identified the visual areas that responded significantly more strongly $(P<.05$, corrected) to the rotating polar stimulus than to the blank background stimulation. Statistical maps were calculated by correlating the signal time course with a reference function for each voxel based on known hemodynamic response properties of the BOLD signal $[17,18]$. The borders of the visual areas of interest $(\mathrm{V} 1, \mathrm{~V} 2 / \mathrm{V} 3, \mathrm{~V} 4, \mathrm{MT})$ were identified based on anatomical criteria [19-22] and regions of interest identified that contained all activated voxels $(P<.05$, corrected) within the borders of these areas.

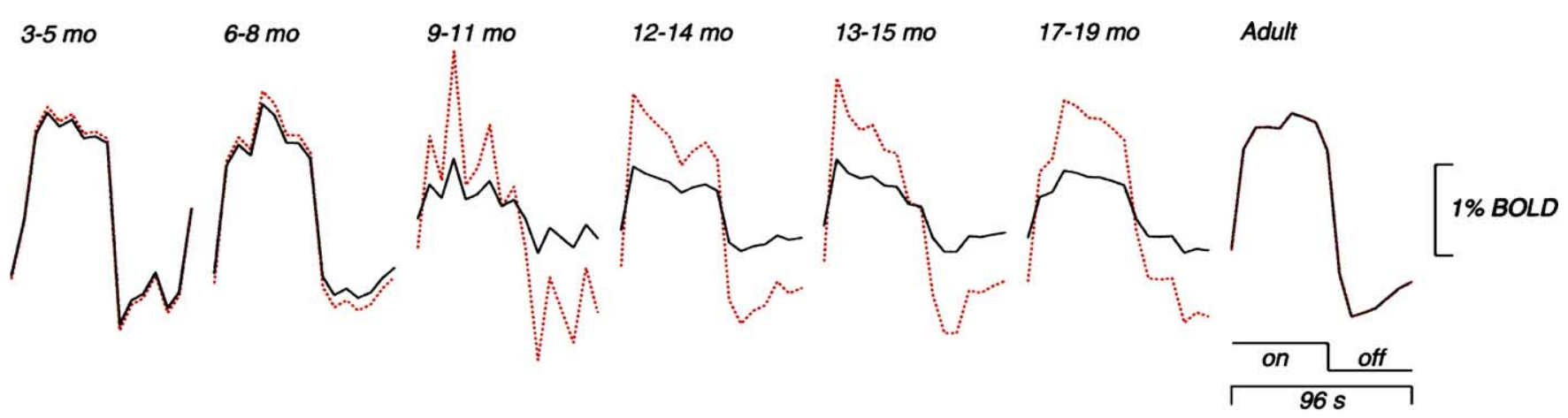

Fig. 4. Averaged BOLD traces showing visual activation in area V1 in two infant monkeys studied longitudinally and three adult monkeys. Each trace is the average of a number of scanning runs conducted on animals in the indicated age ranges; the responses to the four cycles of the stimulus have been averaged to create a single-cycle response. The solid black traces show the actual percent BOLD signal; the dashed red traces have been scaled so that each trace has equal power to facilitate comparison of the response waveform shapes. 


\section{Results}

Significant activations were obtained at even the youngest ages studied (3-5 months). Fig. 2 shows activation patterns for the two infant monkeys at two ages. Significantly stronger activations $(P<.05$, corrected) were recorded to the rotating polar stimulus than the blank background stimulation in the retinotopic visual areas. Fig. 3 shows activation maps for the same stimulus in the retinotopic areas for an adult monkey tested at two different ages $(4.9,6.8$ years). Similar results were observed at other

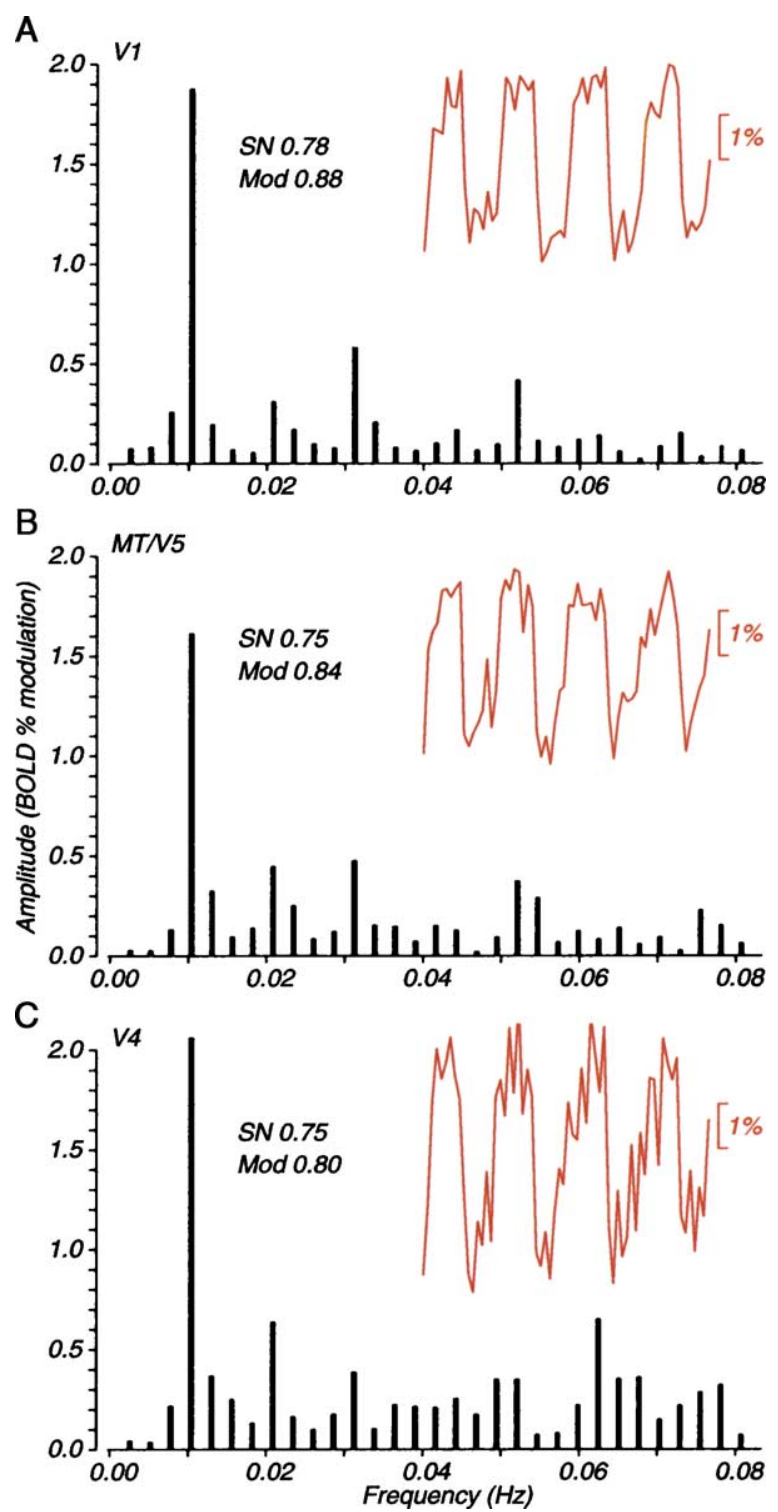

Fig. 5. Frequency-domain analysis of visual activation in three visual areas in an adult monkey. Each panel shows the signal recorded from V1 (A), MT/V5 (B) and V4 (C). Within each panel, the red inset trace shows the time course of the BOLD signal for the defined region of interest; the 384-s stimulus period contained four cycles, each containing $48 \mathrm{~s}$ of patterned visual stimulation and $48 \mathrm{~s}$ of blank. The histogram shows the DFT amplitude spectrum of the red trace - the stimulus frequency is $0.0104 \mathrm{~Hz}$. The values SN and Mod give two figures of merit for each response that are described in the text.
A
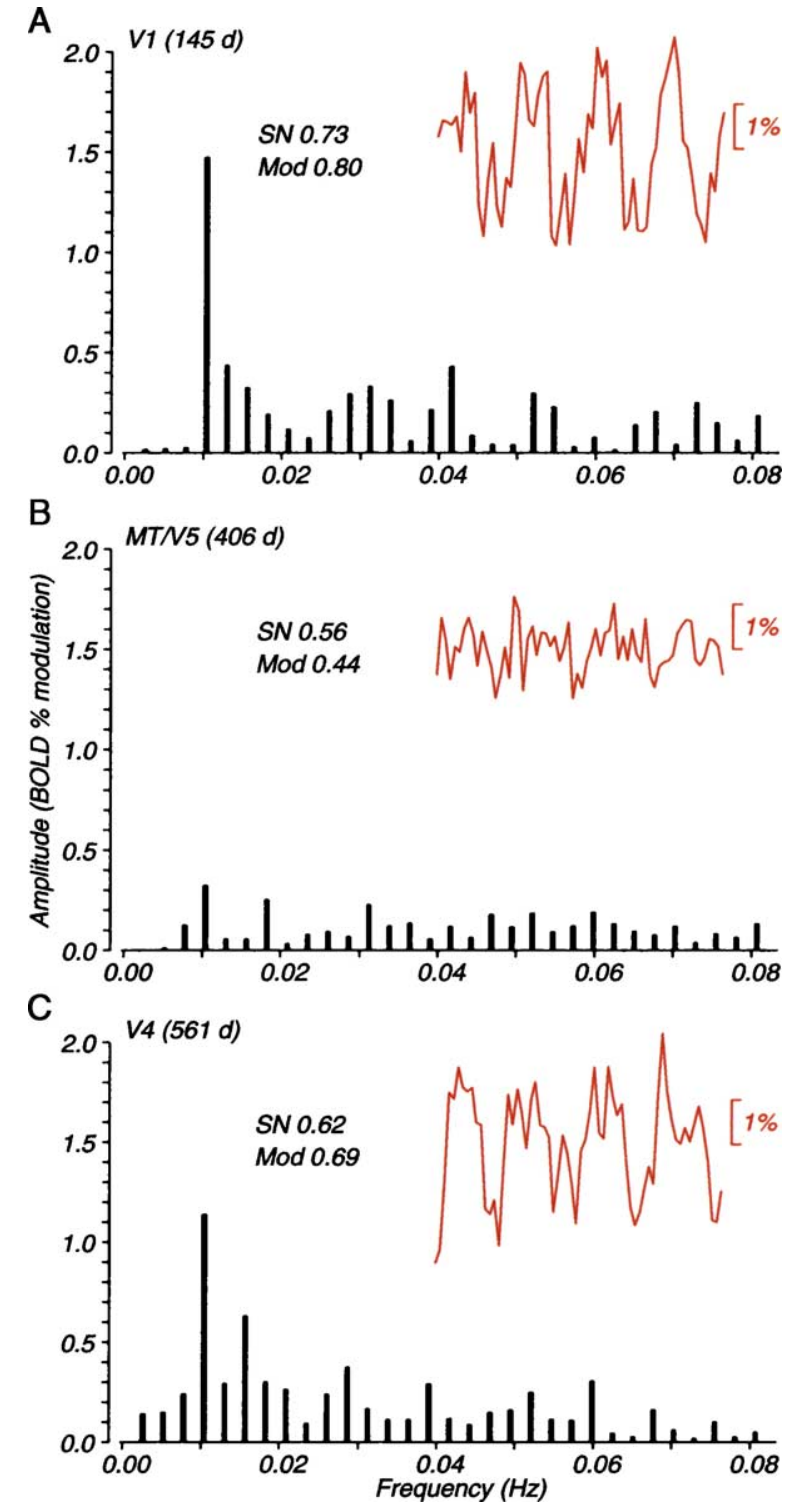

Fig. 6. Frequency-domain analysis of visual activation in three visual areas in infant monkeys; format as in Fig. 4. The data for V1 (A) were obtained at 145 days, for MT/V5 (B) at 406 days, and for V4 (C) at 561 days.

ages for the infant monkeys and in two more adult monkeys. At first glance, it appears that activations in V4 and MT are evident only at later ages in both infant monkeys. Yet, a lack of activation in these regions can be also observed in adult monkeys, as it is shown Fig. 3 (top) in a scan of an animal 4.9 years old. Over the last 8 years in more than 1000 scans, in which responses to the same polar-transformed checkerboards were tested in more than 50 monkeys, activation of the area V4 was evident in most and that of MT in the vast majority of the sessions. Due to the small number of infant monkeys tested in this study, and the lack of rigorous collective statistical analysis of our adult monkey data, however, we cannot exclude the possibility that the differences in the activation maps across scanning sessions reflect differences in the physiological condition of the animals 


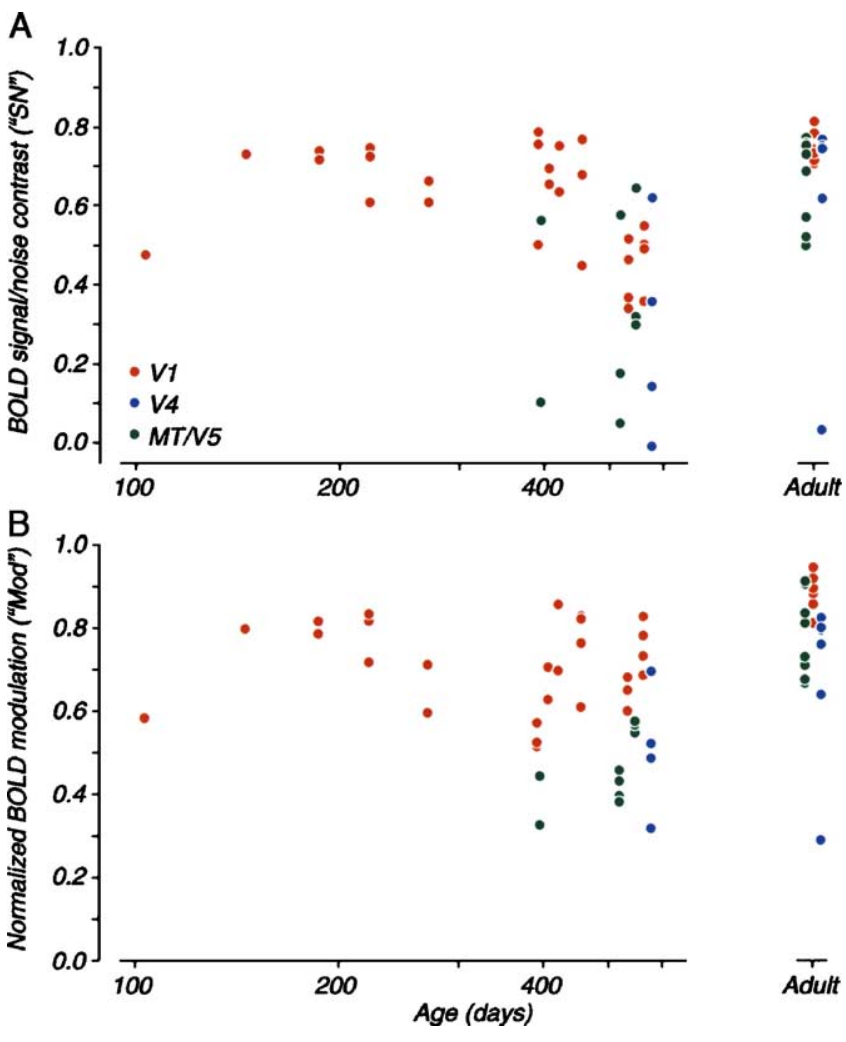

Fig. 7. Development of visual activation in three visual areas, V1 (red), V4 (blue) and MT/V5 (green). Data for V4 and MT/V5 have been offset slightly for clarity. (A) Development measured using a contrast measure of SNR, computed by taking the ratio of modulation at the stimulus frequency to the mean of the two adjacent frequencies in the DFT. (B) Development measured using a normalized measure of BOLD modulation at the stimulus frequency, computed by taking the ratio of modulation at the stimulus frequency to the square root of the total spectral power. See text for details.

under anesthesia that may have stronger effects on the activation of higher extrastriate areas (e.g., V4, MT).

To further evaluate the activations for visual patterns across age, we analyzed the time courses of activation in all significantly activated voxels in areas V1, V4 and MT/ V5. For each scanning session, we computed the time course of the fMRI response by averaging the data from all the activated voxels within these regions. Area V1 was activated in a robust and reliable manner in all scanning sessions for both the infant and adult monkeys. Fig. 4 shows the time courses in V1 for different ages averaged for the two infant monkeys and the time courses averaged across the scanning sessions for the adult animals. There is evident and robust visual activation of $\mathrm{V} 1$ even in the youngest animals, with BOLD signal excursions in excess of $2 \%$. Curiously, the amplitude of the BOLD signal then appears to decrease, dropping below $1 \%$ on average for animals aged 9-19 months. For the adult animals, the signal was again large and robust. In part, the explanation of this reduction may lie in the fact that the overall recorded BOLD signal power measured at those ages was found to be lower.
To provide a quantitative basis for comparison of visual activation in animals of different ages despite the variations in the overall BOLD amplitude evident in Fig. 4, we used a frequency-domain analysis of the BOLD signal. Our reasoning was that a number of factors might contribute to changes in the absolute magnitude of the BOLD signal with age, but that these factors should act equally on visually activated and background components of the signal. The results of this analysis are shown in Figs. 5 and 6. Each part of these figures shows the time course of the BOLD signal across the $384 \mathrm{~s}$ of the trial (shown in red in units of percent modulation) and the amplitude spectrum of this signal computed from a DFT (shown in black bars). Because the stimulus contained four cycles of pattern and blank, the fundamental stimulus frequency corresponds to the fourth harmonic of the entire trial, or $0.0104 \mathrm{~Hz}$. We used two methods to compute a figure of merit for these responses. The first, signal/noise contrast, takes the relationship of the spectrum amplitude at the fundamental stimulus frequency to the mean amplitude at the two flanking frequencies (which are not harmonically related to the stimulus frequency). To normalize this relationship we take a contrast measure as

$\mathrm{SN}=\frac{\left[a_{\omega}-0.5\left(a_{\omega-}+a_{\omega+}\right)\right]}{\left[a_{\omega}+0.5\left(a_{\omega-}+a_{\omega+}\right)\right]}$

where $a_{\omega}$ is the BOLD amplitude at the fundamental frequency, and $a_{\omega-}$ and $a_{\omega+}$ are the amplitudes at the adjacent frequencies. The second, normalized BOLD modulation, is defined as

$\operatorname{Mod}=a_{\omega} / \sqrt{\sum_{i} a_{i}^{2}}$

where $a_{i}$ is the amplitude at frequency $i$.

Fig. 5 shows this analysis for three regions of interest for a single scanning run in an adult monkey. The regions of interest are defined in V1 (A), V5/MT (B), and V4 (C). The BOLD traces show strong synchronized activation in each area, and the spectra show corresponding peaks at the stimulus frequency and its first few harmonics. The SN and Mod measures for all three cases approach 1, indicating strong visual driving of the BOLD signal.

Fig. 6 shows a similar analysis for three regions of interest for three scanning sessions in the infant monkeys. Fig. 6A shows robust visual activation in V1 measured at the age of 145 days. Fig. 6B shows somewhat weaker, but consistent activation in MT/V5 measured at 406 days. Fig. 6C shows activation in V4 measured at 561 days. The values of SN and Mod in Figs. 6B and 6C are substantially lower than those in the corresponding adult cases (Figs. 5B and 5C).

As suggested by Fig. 4, and by the individual traces in Figs. 5A and 6A, we found strong visual activation in V1 in monkeys of all ages. In V5/MT and V4, however, we did not 
find sufficiently reliable activation to quantify in the frequency-domain analysis in the younger animals. To visualize these trends, Fig. 7 shows the developmental course of the SN and Mod measures (Fig. 7A and 7B) for all three visual areas (red, blue, and green symbols). The developmental progression of the $\mathrm{SN}$ measure shown in Fig. 7A is basically flat for V1 (red), but the onset of response is delayed and the first-measured responses are relatively noisy in V4 and MT/V5 (blue, green). The Mod measure shows a somewhat more graded development, in which there seems to be a gradual improvement in the measure with time in V1 (red) and more rapid improvements after delayed onset in V4 and MT/V5 (blue, green).

The difference between the developmental course of SN and Mod appears to be due, as suggested by inspection of the examples in Figs. 5 and 6, to a relative increase in the BOLD activation at low frequencies near the fundamental compared to higher frequencies. This suggests that it is BOLD noise - whose origin might be neural, neurovascular or vascular - that contributes to the delayed emergence of signals from extrastriate cortical areas.

\section{Discussion}

The most prominent result of the present study is that it is possible to image visually evoked activity using standard fMRI techniques in monkeys as young as 103 days. Given the strength of the responses we observed in V1 of even the youngest animals we tested, there is every reason to believe that the BOLD fMRI technique could be used to study visual development at even earlier ages. Our results also show what appears to be a cascade of development, with activation in extrastriate areas downstream of V1 emerging only later in development. Obviously, an important question is whether the differences we report here reflect the maturation of the neural or the vascular system.

Evidently, several issues arise when attempting to compare neural activations of infants - whether of monkeys or humans - with those of adults, including the concordance of functional maps, and the magnitude, latency and time course of the hemodynamic responses themselves. Over the last few years, a small number of studies begun to address the confound of intergroup vascular differences by comparing the hemodynamic responses of children and adults during sensory stimulation or motor tasks [23-25]. These studies reported minor or no differences in the time course or locations of activations; yet, the use of stimulation or motor tasks confounds disparity in cognitive control and performance with possible differences in vascular reactivity and dynamics.

Vascular challenges, such as hypoxia and hypercapnia, are alternatives to sensory stimulation by eliciting hemodynamic responses that are not derived from modulation of neuronal activity alone. Hypercapnia was initially introduced in order to remove the effects of changes in cerebral blood flow $(\mathrm{CBF})$ and cerebral blood volume from those due to neural activation and its subsequent increases in cerebral metabolic rate of oxygen $\left(\mathrm{CMRO}_{2}\right)$; in other words, in order to calibrate the BOLD responses [26]. However, later on it was also used to study differences in vascular reactivity between different individuals, between groups of patients and controls, and groups of adults of different ages (see, e.g., [27-29]). A most recent study provided the first data on differences between children and adults. Thomason et al. [30] examined changes in the BOLD fMRI signal elicited by breath holding $(\mathrm{BH})$ in children (7-10 years) and adults (18-29 years). Differences were found in a number of variables, including the ratio of activated gray to white matter volume, the total volume of activation, the signal intensity, and the signal-to-noise ratio (SNR). Notably, this study reports that responses to $\mathrm{BH}$ in children were systematically characterized by larger signal intensity and increased noise [30].

Our findings of change in the amplitude of stimulusinduced modulation and in the normalized BOLD modulation as depicted in Fig. 7B are consistent with those reported in children in the aforementioned study. The origin of increased intensity and reduced SNR is currently not known. One can hypothesize that $\mathrm{CMRO}_{2}$ is significantly different in infants and adults, with the former having a higher rate, which results to responses of greater amplitude. An increased oxygen extraction fraction would add on to this effect. Alternatively, infants may have a greater number of activated cells per voxel. Given the strong dependence of BOLD on perisynaptic processes [31], one would then expect an increase in $\mathrm{CMRO}_{2}, \mathrm{CBF}$ and BOLD activation. Decreases in different bands of EEG with age have been indeed described for children [32,33]. In the case of such an electrical activity-based hypothesis, one can further speculate that maturational processes, such as synaptic pruning and increases of response selectivity, may reduce stimulusinduced neural activation and its subsequent BOLD response. Increased response selectivity may also underlie the reduction in noise that yields the increase in normalized BOLD modulation in Fig. 7B.

In the infants, no session prior to about 14 months produced significant activation in the areas V4 and MT/V5. The apparent late development of activation in extrastriate visual areas could be accounted for by any of the hypotheses mentioned above, but it is not without precedent. Distler et al. [9] found delayed maturation of dorsal stream areas relative to V1 using a metabolic assay. Similarly, Rodman et al. [4] provide physiological evidence for relatively late development of neuronal responsiveness in MT/V5. Further, inferotemporal areas became physiologically responsive several months after the dorsal stream areas. However, these studies all found reliable visually driven neural activity in extrastriate areas by 6-8 months after birth, somewhat earlier than we find with fMRI. Moreover, Movshon et al. [7] found robust responses from infant neurons in areas MT/V5 during single unit recording under similar anesthetic conditions to those used in the current study. The predominant difference 
between infant and adult responses at the single unit level was extraordinarily long latency to first spike in infant neurones. The source of the long latency is unclear. However, the slow, potentially incoherent responses from these neurones could render the population signal indistinguishable from noise and thus result in decreased BOLD contrast effects.

In summary, the reliable visually evoked activation observed in V1 across all ages rules out a general failure of functional imaging as a method for studying visual development. Our demonstration of the feasibility of functional imaging in young infant monkeys provides the basis for future studies comparing the development of visual behavior with that of brain organization in individual animals. Further studies, perhaps combining imaging and physiology, will be needed to provide definitive evidence for a cascade of development within the visual pathways. Continued development of this technology could provide the opportunity to image neural activity simultaneously with behavior in awake infant monkeys, thus allowing studies of cognitive or motor function.

\section{Acknowledgments}

This work was supported by the Max Planck Society and by NIH project grants EY2017 to JAM and EY5864 to LK. We express our gratitude to Stefan Weber for his creative technical help, and to Dr. Beatriz Haro-Gandara, Dr. Heinz Guggenberger, Marcel Henni, Jan Laucken, Kathrin Guhr, Emica Coric, Arne Deubelius and Pegah Sarkheil for their assistance with the experiments and with the rearing of the infant monkeys.

\section{References}

[1] Nelson CA, Luciana M. Handbook of developmental cognitive neuroscience. Cambridge: MIT Press; 2001.

[2] Daw N. Visual development. New York: Plenum Press; 1995.

[3] Kiorpes L, Movshon JA. Neural limitations on visual development in primates. In: Chalupa LM, Werner JS, editors. The visual neurosciences. Cambridge: MIT Press; 2003 [Chapter 12]. p. 159-73.

[4] Rodman HR, Scalaidhe SP, Gross CG. Response properties of neurones in temporal cortical visual areas of infant monkeys. J Neurophysiol 1993;70:1115-36.

[5] Chino YM, Smith III EL, Hatta S, Cheng H. Postnatal development of binocular disparity sensitivity in neurons of the primate visual cortex. J Neurosci 1997;17:296-307.

[6] Movshon JA, Kiorpes L, Cavanaugh JR, Hawken MJ. Receptive field properties and surround interactions in V1 neurons in infant macaque monkeys. Society for Neuroscience Abstracts 1999;25:1048.

[7] Movshon JA, Rust NC, Kohn A, Kiorpes L, Hawken MJ. Receptive field properties of MT neurons in infant macaques. Society for Neuroscience Abstracts 2003;126.8.

[8] Zhang B, Zheng J, Watanabe I, Maruko I, Bi H, Smith III EL, et al. Delayed maturation of receptive field center/surround mechanisms in V2. Proc Natl Acad Sci U S A 2005;102:5862-7.

[9] Distler C, Bachevalier J, Kennedy C, Mishkin M, Ungerleider LG. Functional development of the corticocortical pathway for motion analysis in the macaque monkey: a ${ }^{14} \mathrm{C}-2$-deoxyglucose study. Cereb Cortex 1996;6:184-95.
[10] Ungerleider LG, Pasternak T. Ventral and dorsal cortical processing streams. In: Chalupa LM, Werner JS Editors. The visual neurosciences. Cambridge: MIT Press; 2003 [Chapter 34]. p. 541-62.

[11] Erberich SG, Friedlich P, Seri I, Nelson MD, Bluml S. Functional MRI in neonates using neonatal head coil and MR compatible incubator. Neuroimage 2003;20:683-92.

[12] Erberich SG, Panigraphy A, Friedlich P, Seri I, Nelson MD, Gilles F. Somatosensory lateralization in the newborn brain. Neuroimage 2005 2006;29:155-61.

[13] Kiorpes L. The development of vernier acuity and grating acuity in normally-reared monkeys. Vis Neurosci 1992;9:243-51.

[14] Kiorpes L, Movshon JA. Development of sensitivity to visual motion in macaque monkeys. Vis Neurosci 2004;21:851-9.

[15] Kiorpes L, Bassin SA. Development of contour integration in macaque monkeys. Vis Neurosci 2003;20:567-75.

[16] Logothetis NK, Guggenberger H, Peled S, Pauls J. Functional imaging of the monkey brain. Nat Neurosci 1999;2:555-62.

[17] Boynton GM, Engel SA, Glover GH, Heeger DJ. Linear systems analysis of functional magnetic resonance imaging in human V1. J Neurosci 1996;16:4207-21.

[18] Cohen MS. Parametric analysis of fMRI data using linear systems methods. Neuroimage 1997;6:93-103.

[19] Gattass R, Gross CG, Sandell JH. Visual topography of V2 in the macaque. J Comp Neurol 1981;201:519-39.

[20] Desimone R, Ungerleider LG. Multiple visual areas in the caudal superior temporal sulcus of the macaque. J Comp Neurol 1986;248: $164-89$.

[21] Gattass R, Sousa AP, Gross CG. Visuotopic organization and extent of V3 and V4 of the macaque. J Neurosci 1988;8:1831-45.

[22] Brewer AA, Press WA, Logothetis NK, Wandell BA. Visual areas in macaque cortex measured using functional magnetic resonance imaging. J Neurosci 2002;22:10416-26.

[23] Kang HC, Burgund ED, Lugar HM, Petersen SE, Schlaggar BL. Comparison of functional activation foci in children and adults using a common stereotactic space. Neuroimage 2001;19:16-28.

[24] Richter W, Richter M. The shape of the fMRI BOLD response in children and adults changes systematically with age. Neuroimage 2002;20:1122-31.

[25] Marcar VL, Strassle AE, Loenneker T, Schwarz U, Martin E. The influence of cortical maturation on the BOLD response: an fMRI study of visual cortex in children. Pediatr Res 2004;56: 967-74.

[26] Bandettini PA, Wong EC. A hypercapnia-based normalization method for improved spatial localization of human brain activation with fMRI. NMR Biomed 1997;10:197-203.

[27] Kastrup A, Dichgans J, Niemeier M, Schabet M. Changes of cerebrovascular CO2 reactivity during normal aging. Stroke 1998; 29:1311-4.

[28] Kastrup A, Li TQ, Takahashi A, Glover GH, Moseley ME. Functional magnetic resonance imaging of regional cerebral blood oxygenation changes during breath holding. Stroke 1998;29:2641-5.

[29] Corfield DR, Murphy K, Josephs O, Adams L, Turner R. Does hypercapnia-induced cerebral vasodilation modulate the hemodynamic response to neural activation? Neuroimage 2001;13:1207-11.

[30] Thomason ME, Burrows BE, Gabrieli JD, Glover GH. Breath holding reveals differences in fMRI BOLD signal in children and adults. Neuroimage 2005;25:824-37.

[31] Logothetis NK, Pauls J, Augath M, Trinath T, Oeltermann A. Neurophysiological investigation of the basis of the fMRI signal. Nature 2001;412:150-7.

[32] Wada M, Ogawa T, Sonoda H, Sato K. Development of relative power contribution ratio of the EEG in normal children: a multivariate autoregressive modeling approach. Electroencephalogr Clin Neurophysiol 1996;98:69-75.

[33] Gasser T, Verleger R, Bacher P, Sroka L. Development of the EEG of school-age children and adolescents. I. Analysis of band power. Electroencephalogr Clin Neurophysiol 1988;69:91-9. 